\title{
Modified dispersion law for spin waves coupled to a superconductor
}

Cite as: J. Appl. Phys. 124, 233903 (2018); https://doi.org/10.1063/1.5077086

Submitted: 24 October 2018 . Accepted: 04 December 2018 . Published Online: 20 December 2018

I. A. Golovchanskiy (D), N. N. Abramov, V. S. Stolyarov (D), V. V. Ryazanov, A. A. Golubov, and A. V. Ustinov
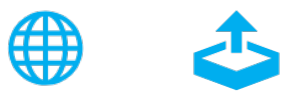

\section{ARTICLES YOU MAY BE INTERESTED IN}

Linear and nonlinear spin-wave dynamics in ultralow-damping microstructured $\mathrm{CO}_{2} \mathrm{FeAl}$ Heusler waveguide

Applied Physics Letters 113, 232404 (2018); https://doi.org/10.1063/1.5038836

Tutorial: An introduction to terahertz time domain spectroscopy (THz-TDS)

Journal of Applied Physics 124, 231101 (2018); https://doi.org/10.1063/1.5047659

Magnified photonic spin-Hall effect with curved hyperbolic metamaterials

Journal of Applied Physics 124, 233104 (2018); https://doi.org/10.1063/1.5078634

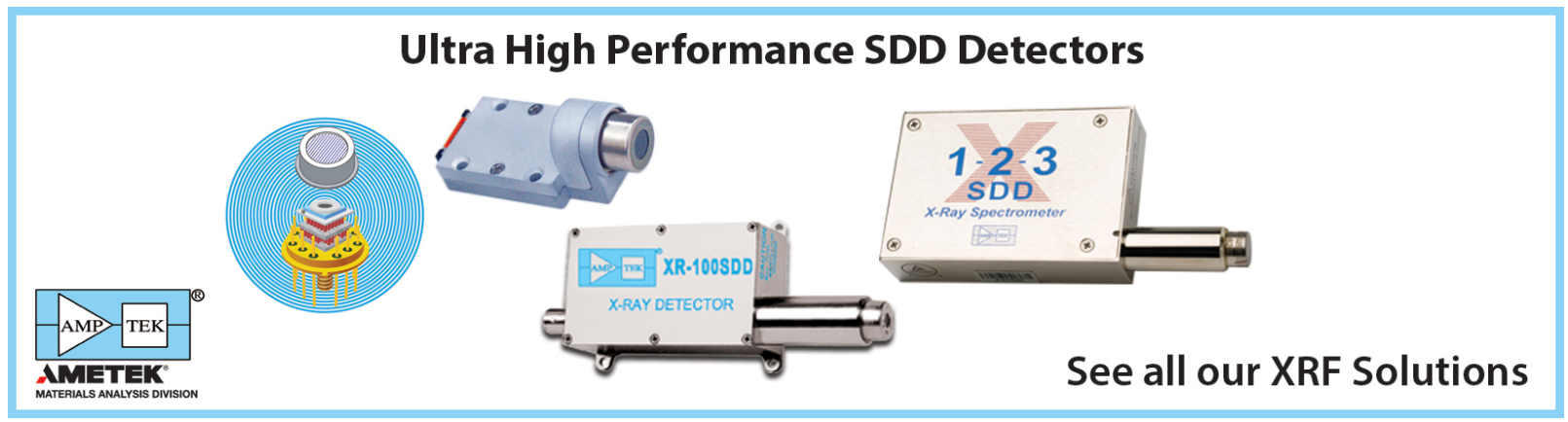




\title{
Modified dispersion law for spin waves coupled to a superconductor
}

\author{
I. A. Golovchanskiy, ${ }^{1,2}$ N. N. Abramov, ${ }^{2}$ V. S. Stolyarov, ${ }^{1,3,4}$ V. V. Ryazanov, ${ }^{2,3,4}$ \\ A. A. Golubov, ${ }^{1,5}$ and A. V. Ustinov ${ }^{2,6}$ \\ ${ }^{1}$ Moscow Institute of Physics and Technology, State University, 9 Institutskiy per., Dolgoprudny, \\ Moscow Region 141700, Russia \\ ${ }^{2}$ The Laboratory of Superconducting Metamaterials, National University of Science and Technology MISIS, \\ 4 Leninsky prosp., Moscow 119049, Russia \\ ${ }^{3}$ Institute of Solid State Physics (ISSP RAS), Chernogolovka, Moscow Region 142432, Russia \\ ${ }^{4}$ Solid State Physics Department, Kazan Federal University, Kazan 420008, Russia \\ ${ }^{5}$ Faculty of Science and Technology and MESA+ Institute for Nanotechnology, University of Twente, 7500 AE \\ Enschede, The Netherlands \\ ${ }^{6}$ Physikalisches Institut, Karlsruhe Institute of Technology, 76131 Karlsruhe, Germany
}

(Received 24 October 2018; accepted 4 December 2018; published online 20 December 2018)

\begin{abstract}
In this work, we consider dispersion laws of spin waves that propagate in a ferromagnet/superconductor bilayer, specifically in a ferromagnetic film coupled inductively to a superconductor. The coupling is viewed as an interaction of a spin wave in a ferromagnetic film with its mirrored image generated by the superconductor. We show that, in general, the coupling enhances substantially the phase velocity of magnons in in-plane spin wave geometries. In addition, a heavy nonreciprocity of the dispersion law is observed in the magnetostatic surface spin wave geometry where the phase velocity depends on the direction of the wave propagation. Published by AIP Publishing. https://doi.org/10.1063/1.5077086
\end{abstract}

\section{INTRODUCTION}

Spin waves or magnons are eigen-disturbances in magnetic moments propagating within a magnetic material, such as a ferromagnet (FM), ferrimagnet, or antiferromagnet, via exchange or magnetostatic interactions. While spin waves have been known for more than 60 years, ${ }^{1,2}$ nowadays, an interest in magnonics has re-emerged due to a rapid advancement of nanotechnology and due to the development of new experimental techniques for studying high-frequency magnetization dynamics. In particular, a good overview of modern trends in magnonics can be found in the Special Issue on Magnonics, Journal of Physics D: Applied Physics 2017. Also, a few good books have been released dedicated to magnonics. ${ }^{3-5}$

Despite the challenges for magnonics, ${ }^{6}$ including poor conversion efficiency of electromagnetic to spin wave signal and rapid attenuation of spin wave signals, spin waves are particularly promising for information transfer and processing owing to the following advantages: (i) A dispersion law of a spin-wave can be easily adjusted simply by changing the applied magnetic field, thickness, and geometry of the media. (ii) Spin waves enable information transfer across long distances ${ }^{7}$ with small dissipation, due to the charge-current-free nature at high microwave operational speed, and with low power consumption. (iii) The wavelength of a spin wave is in a micro- and sub-micro-scale at microwave frequencies which allows for the building of an actual magnonic micro-device for processing of microwave information. (iv) Finally, unlike photons, magnons propagate in magnetically ordered (magnonic) media only and do not couple parasitically to a non-magnetic surrounding. These advantages favor the development of magnonics toward information transfer and processing, ${ }^{6,8,9}$ including quantum information, ${ }^{10,11}$ for engineering of spin wave logic devices, ${ }^{12-14}$ and periodic magnonic bandgap structures. ${ }^{15,16}$

Recently, it has been noted that once a FM film is placed on a superconducting (SC) surface, the dispersion law of magnons is modified drastically. In Ref. 17, it has been demonstrated both experimentally and numerically that the phase velocity of magnons in a permalloy film increases when the FM film is placed on and inductively coupled to a superconducting surface. The effect emerges due to the magnetostatic interaction of a spin wave with its mirrored image produced by the screening currents of a superconductor in the ideal diamagnetic Meissner state. In this work, we explore this effect on the dispersion law of magnons in in-plane spin wave geometries, namely, magnetostatic surface spin wave (MSSW) and backward volume spin wave (BVSW) modes, using the micromagnetic simulations ${ }^{18,19}$ combined with a micromagnetic method of images. ${ }^{17}$

\section{SPIN WAVE MODES: SIMULATION DETAILS}

Based on the orientation of the spin wave propagation $(\vec{k})$ relative to the static magnetization $(\vec{M})$, there are three well known modes of magnetostatic spin waves: $:^{2,3,15,20,21}$ the magnetostatic surface spin wave mode, the backward volume spin wave mode, and also the forward volume spin wave (FVSW) mode.

The MSSW mode is observed when the in-plane wave vector is oriented perpendicular to the direction of magnetization $(\vec{k} \perp \vec{M})$ and follows the dispersion law

$$
\begin{aligned}
\left(2 \pi f / \mu_{0} \gamma\right)^{2}= & \left(H+H_{a}\right)\left(H+H_{a}+M_{s}\right) \\
& +M_{s}^{2}[1-\exp (-2 k d)] / 4,
\end{aligned}
$$

where $f$ is the wave frequency, $\mu_{0}$ is the vacuum permeability, $\gamma$ is the gyromagnetic ratio, $k=2 \pi / \lambda$ is the wave vector, $\lambda$ is the wavelength, $H$ is the applied magnetic field, $H_{a}$ is the anizotropy field, $M_{s}$ is the saturation magnetization, and $d$ is the thickness of the ferromagnetic film.

The BVSW mode is observed when the in-plane wave vector is aligned with the direction of in-plane magnetization 
$(\vec{k} \| \vec{M})$ and follows a different dispersion law

$$
\begin{aligned}
\left(2 \pi f / \mu_{0} \gamma\right)^{2}= & \left(H+H_{a}\right) \\
& \times\left\{H+H_{a}+M_{s}[1-\exp (-k d)] / k d\right\} .
\end{aligned}
$$

The BVSW mode is often of specific interest due to its negative group velocity. ${ }^{3,21,22}$

If a spin wave is excited with short wavelength comparable with the exchange length $\left(2 A / \mu_{0} M_{s}^{2}\right)^{1 / 2}$, where $A$ is the exchange stiffness constant, the exchange energy becomes substantial or even dominant, and the magnetostatic spin wave dispersion law is supplemented by the effective exchange field: in Eqs. (1) and (2), the magnetic field $H$ is substituted by $H+\left(2 A / \mu_{0} M_{s}\right) k^{2}$. Note that while exchangedominated spin waves are the most promising for applications owing to a wide range of its high eigen frequencies, these are the hardest to excite due to a sub-micro scale of their typical wavelengths. Operation with exchange-dominated spin waves requires either precise nanotechnology ${ }^{23-25}$ or more sophisticated approaches. ${ }^{26,27}$

The dispersion relation of a ferromagnetic system can be studied numerically using micromagnetic simulations ${ }^{18,19}$ as demonstrated in Refs. 5, 28, and 29. Briefly, the numerical procedure is as follows. A magnetized ferromagnetic film is placed at an applied magnetic field $H$. A magnetic field pulse of a sinc temporal profile is applied locally orthogonally to $\vec{H}$, and the evolution of local magnetic moments in the film $\vec{M}(\vec{r}, t)$ is recorded. The maximum of the amplitude of the space-time Fourier transform of $\vec{M}(\vec{r}, t)$ provides the dispersion $f(\vec{k})$. In order to avoid the reflections, an exponential Gilbert damping profile is set near the boundaries of the film. In this work, a particular MSSW mode and a particular BVSW mode are considered, and therefore, it is sufficient to perform the micromagnetic simulation employing a 1D mesh, following Refs. 5 and 20, in order to capture correctly the magnetostatic spin wave activity.

If a magnetic moment is placed in close proximity to a superconductor, the effective field that acts on a moment changes. The Meissner currents emerge in a superconductor that screen the magnetostatic stray fields of the moment, i.e., the ideal diamagnetic Meissner state is realized. These screening currents affect the actual field acting on a magnetic moment.

The magnetostatic problem of a ferromagnetic film placed on the superconducting surface can be treated with the method of images as magnetostatic interaction of two ferromagnets. ${ }^{17}$ The method of images, illustrated in Fig. 1, implies inductive coupling of macro-spins in the ferromagnetic layer $\vec{M}(x, y, z)=\left(M_{x}, M_{y}, M_{z}\right)$ located over a distance $z$ above the superconducting surface $x-y$ with their mirror images $\vec{M}_{i m}(x, y,-z)=\left(M_{x}, M_{y},-M_{z}\right)$. Numerical implementation of the method of images in micromagnetic simulations is executed by inclusion of all image macro-spins $\vec{M}_{i m}$ for calculation of the dipole-dipole component of the effective field at each time step of integration of the Landau-Lifshitz-Gilbert equation.

In this work, for investigation of magnetostatic modes, $L \times W \times d$ rectangular permalloy FM films are placed in the $x-y$ plane. The films are $1 \mathrm{D}$-meshed to $1 \times 5000 \times 1$ cells

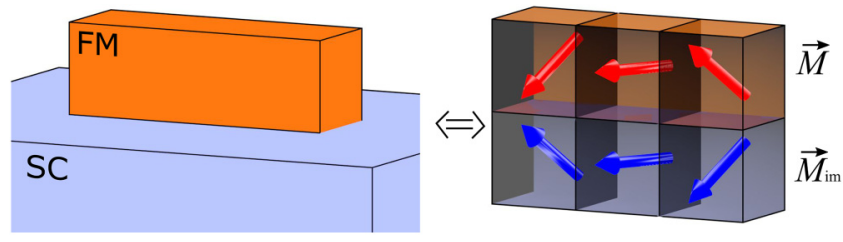

FIG. 1. Illustration of the method of images. Left image: A finite-size ferromagnet (shown in orange) is placed on the surface of a superconductor (shown in blue). The superconductor, as an ideal diamagnet, excludes all magnetostatic stray fields induced by any orientation of spins in the ferromagnet. Right image: Within micromagnetic terms, such coupling is equivalent to the interaction of ferromagnetic macro-spins $\vec{M}$ (red arrows) and their mirrored images $\vec{M}_{i m}$ with respect to the superconducting surface (blue arrows).

along $y$-axis. The magnetic field was applied along the $x$-axis for excitation of the MSSW mode and along the $y$-axis for excitation of the BVSW mode, and the sinc field pulse was applied along the $z$-axis. The following micromagnetic parameters, typical for permalloy films, ${ }^{17,30}$ have been used for simulations: $M_{s}=9.3 \times 10^{5} \mathrm{~A} / \mathrm{m}, H_{a}=2.5 \times 10^{3} \mathrm{~A} / \mathrm{m}$, aligned along $\vec{H}$, the exchange stiffness constant $A=1.3 \times$ $10^{-11} \mathrm{~J} / \mathrm{m}$, and $\gamma=1.856 \times 10^{11} \mathrm{~Hz} / \mathrm{T}$. For investigation of magnetostatic modes, the excitation pulse was of a sinc temporal profile with $f_{\max }$ frequency, of a Gaussian spatial profile with the width $\sigma$, and of an amplitude of $0.001 M_{s}$.

Only in-plane modes are considered in this work, since the out-of-plane FVSW geometry typically requires a high magnetic field $H>M_{s}$ for appropriate excitation of spin waves. At high magnetic field, Abrikosov vortices penetrate the superconductor, which reduces the screening capabilities. In particular, for infinite superconducting thin film at outof-plane magnetic field $B \approx H$, and technically, no screening of magnetic field takes place. Yet, superconductivity persists at $H$ below the second critical field. Therefore, screening capabilities of a superconducting film and applicability of the method of images with its micromagnetic representation (Fig. 1) are not obvious.

\section{RESULTS AND DISCUSSION}

In Fig. 2, we show dispersions for films at $H<<M_{s}$ where dispersions are the steepest. Dispersion laws for several thicknesses and magnetic fields are compared in the supplementary material. Figure 2(a) shows MSSW dispersions simulated for the $L \times W \times d=250 \times 250 \times 0.1 \mu \mathrm{m}^{3}$ film at $H / M_{s}=3.3 \times 10^{-3}, \sigma=100 \mathrm{~nm}$, and $f_{\max }=50 \mathrm{GHz}$. Black solid lines show the dispersion of the plain ferromagnetic film, i.e., with no superconductor in vicinity, obtained with micromagnetic simulation. Blue dashed lines in Fig. 2(a) show the dispersion of the plain ferromagnetic film calculated using the theoretical expression (1). Simulated curves match well the theoretical dispersions indicating applicability of the simulation method.

Red solid curves in Fig. 2(a) show MSSW dispersions of the FM film placed on the SC surface, obtained with micromagnetic simulation combined with the method of images (Fig. 1). It shows that the magnetostatic interaction of MSSW with the perfect diamagnetic surface of the superconductor enhances strongly the phase velocity $f / k$. 

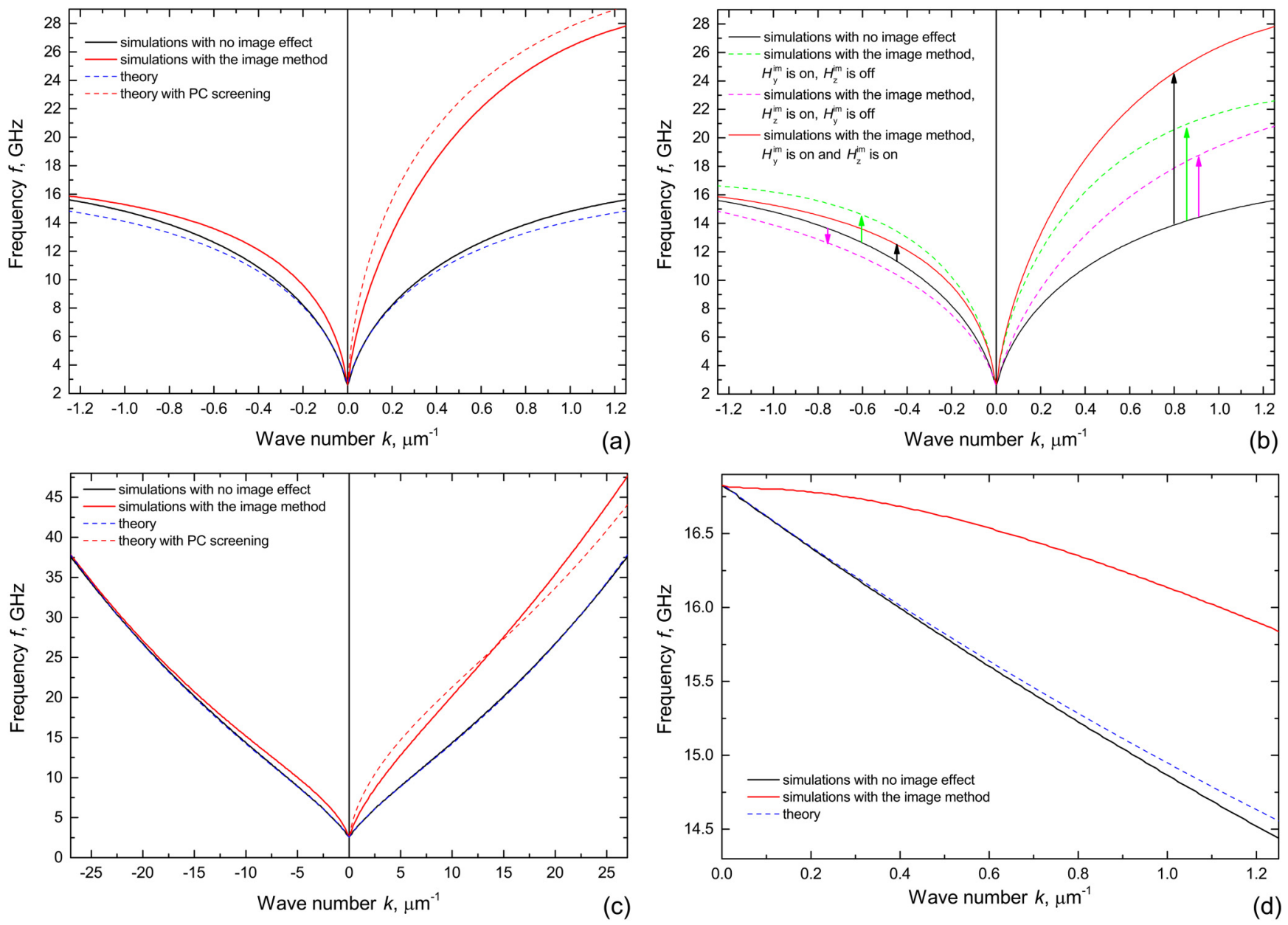

FIG. 2. Dispersion laws for spin waves in different geometries. The dispersions of plain FM films obtained with micromagnetic simulations are shown with solid black curves. The dispersions of plain FM films calculated using theoretical expressions [Eqs. (1) and (2)] are shown with dashed blue curves. The dispersions of FM films on the SC surface obtained with micromagnetic simulations combined with the method of images are shown with solid red curves. (a) Dispersion law for a $L \times W \times d=250 \times 250 \times 0.1 \mu \mathrm{m}^{3}$ film in MSSW geometry derived at $H / M_{s}=3.3 \times 10^{-3}, \sigma=100 \mathrm{~nm}$, and $f_{\text {max }}=50 \mathrm{GHz}$. (b) Dispersion laws for the same case as in (a) but switched on/off AC components of the image magnetostatic stray field. (c) Dispersion law for a $L \times W \times d=15 \times 15 \times 0.003 \mu \mathrm{m}^{3}$ film in MSSW geometry derived at $H / M_{s}=3.3 \times 10^{-3}, \sigma=9 \mathrm{~nm}$, and $f_{\max }=75 \mathrm{GHz}$. (d) Dispersion law for a $L \times W \times d=250 \times 250 \times 0.1 \mu \mathrm{m}^{3}$ film in BVSW geometry derived at $H / M_{s}=2.2 \times 10^{-1}, \sigma=100 \mathrm{~nm}$, and $f_{\max }=50 \mathrm{GHz}$. The theoretical dispersion of the FM film placed on the surface of the perfect conductor for (a) and (c) cases is shown with dashed red curves.

Also, a nonreciprocal behavior is observed: the relative enhancement of the frequency is stronger for positive wave vectors $k$. In particular, for $k=+1 \mu \mathrm{m}^{-1}$, the frequency grows from $f=14.8 \mathrm{GHz}$ to $f=26.4 \mathrm{GHz}$, i.e., almost by a factor of 2 , while for $k=-1 \mu \mathrm{m}^{-1}$, the frequency grows from $f=14.8 \mathrm{GHz}$ only to $f=15.3 \mathrm{GHz}$, i.e., by $3 \%$ only.

The dependence of the dispersion on the direction of the wave-vector $\pm k$, i.e., the nonreciprocity, is a known property of MSSWs. In MSSW geometry, the wave energy is localized at the surface of the film depending on the direction of wave propagation. ${ }^{3,4}$ A change of the direction of the propagation (or alternatively, a change of the direction of the external magnetic field) causes the localization of the MSSW to move to the opposite surface of the film. Any kind of asymmetry of the film or its surrounding causes emerging of the nonreciprocal dispersion relation (see, for instance, Refs. 20 and 31-33). For instance, in MSSW geometry, the frequency bandwidth for positive $k$ is doubled when the FM film is placed on the surface of the perfect conductor $(\mathrm{PC})^{4,31-33}$ as depicted with red dashed curve in Fig. 2(a).
Thus, the nonreciprocity we observe in Fig. 2(a) emerges due to the nonuniform distribution of image magnetostatic fields produced by superconducting Meissner currents. We illustrate it in Fig. 2(b) where we plot dispersions calculated with the same parameters as in Fig. 2(a) but with switched on/off individual $\mathrm{y}$ and $\mathrm{z}$ components of the magnetostatic stray field of the image $H_{y}^{i m}$ and $H_{z}^{i m}$. The image component $H_{y}^{i m}$ operates in phase with the dipole magnetostatic stray self-field component of the film $H_{y}^{d}$ for both $+k$ and $-k$, and the action of $H_{y}^{i m}$ increases the dispersion frequency [depicted with green dashed lines and arrows in Fig. 2(b)]. Yet, $H_{y}^{i m}$ is larger for $+k$ and results in larger frequency gain at $+k$ as compared with the effect at $-k$. In contrast, the image component $H_{z}^{i m}$ operates in-phase with $H_{z}^{d}$ for $+k$ and in opposite phase for $-k$ [depicted with purple dashed lines and arrows in Fig. 2(b)]. Also, the absolute value of $H_{z}^{i m}$ is larger at $+k$ which results in larger frequency difference at $+k$ as compared with the effect at $-k$. The cumulative action of image fields $H_{y}^{i m}$ and $H_{z}^{i m}$ results in substantially larger frequency gain at $+k$ [depicted with red solid lines and 
black arrows in Fig. 2(b)]. At large applied magnetic fields (see the supplementary material), the $H_{y}^{i m}$ and $H_{z}^{i m}$ components are compensated out and the effect at $-k$ is negligible.

Interaction of the exchange spin waves with the superconducting screening currents is considered for much thinner FM film of $d=3 \mathrm{~nm}$, where the dipolar dispersion is suppressed. Figure 2(c) shows the MSSW dispersions for the $L \times W \times d=15 \times 15 \times 0.003 \mu \mathrm{m}^{3}$ film simulated at $H / M_{s}=3.3 \times 10^{-3}, \sigma=9 \mathrm{~nm}$, and $f_{\max }=75 \mathrm{GHz}$. Black solid lines show the dispersion of the plain ferromagnetic film, i.e., with no superconductor in vicinity, obtained with micromagnetic simulation. Blue dashed lines in Fig. 2(c) show the dispersion of the plain ferromagnetic film calculated using the theoretical expression (1). Simulated curves match perfectly theoretical dispersions. Red curves in Fig. 2(c) show simulated dispersions of the FM film placed on the SC surface. It shows that the magnetostatic interaction of the spin wave with the perfect diamagnetic surface of the superconductor enhances the phase velocity even for short spin waves in the exchange regime. The nonreciprocal behavior is also observed. In particular, for $k=+20 \mu \mathrm{m}^{-1}$, the frequency grows from $f=26.6 \mathrm{GHz}$ to $f=35.4 \mathrm{GHz}$, while for $k=-20 \mu \mathrm{m}^{-1}$, the frequency grows from $f=26.6 \mathrm{GHz}$ only to $f=27.1 \mathrm{GHz}$. The simulated dispersion curve is in good agreement with the one calculated for the film coupled to the PC surface [red dashed curve in Fig. 2(c)].

Figure 2(d) shows the BVSW dispersion for $L \times W \times$ $d=250 \times 250 \times 0.1 \mu \mathrm{m}^{3}$ permalloy films simulated at $H / M_{s}=2.2 \times 10^{-1}, \sigma=100 \mathrm{~nm}$, and $f_{\max }=50 \mathrm{GHz}$. Black solid line shows the dispersion of the plain ferromagnetic film, i.e., with no superconductor in vicinity, obtained with micromagnetic simulation. Blue dashed line in Fig. 2(d) shows the dispersion of the plain ferromagnetic film calculated using the theoretical expression (2). Simulated curve matches well the theoretical dispersion. Red curve in Fig. 2(d) shows the simulated BVSW dispersion of the FM film placed on the SC surface. It shows that the magnetostatic interaction of BVSW with the perfect diamagnetic surface of the superconductor also enhances the phase velocity. In addition, coupling with the superconductor changes the curvature of the dispersion law from concave to convex.

As a final remark, we discuss applicability and limitations of the SC screening effect and of the method of images. We presume that the limiting wavelength of a spin wave for screening is comparable with the London penetration depth of a superconductor, which ranges from hundreds of $\mathrm{nm}$ in high temperature superconductors to $10-100 \mathrm{~nm}$ in conventional low temperature superconductors. Small limiting length favorably distinguishes superconductors from the real conductors where the wavelength for screening as the perfect electric conductor is limited by the skin depth ${ }^{31}$ which is in micrometers for the microwave frequency range. The operation frequency for screening of a spin wave by a superconductor is limited by the superconducting gap frequency which is typically in sub-THz range. ${ }^{34}$ We presume that the limiting in-plane magnetic field, i.e., magnetic field aligned along the surface, for effective screening is the second critical field for type two superconductors. Indeed, while above the first critical field magnetic flux penetrates into a superconductor with vortices, the superconducting surface retains its screening capabilities for Oe fields. At an out-of-plane magnetic field above the first critical field, the destructive two magnon scattering ${ }^{35}$ of spin waves on the superconducting vortex lattice may take effect.

\section{CONCLUSION}

In sum, we have numerically considered the effect of the inductive coupling of spin waves with the superconducting surface on the dispersion law of spin waves. The coupling is viewed as the magnetostatic interaction of micromagnetic macro-spins in the ferromagnetic film with their mirrored image generated by screening currents of the superconductor. We have shown that, in general, the coupling substantially enhances the phase velocity of spin waves in any in-plane geometry: MSSW mode, BVSW mode, and in the exchange regime. In addition, a strong nonreciprocity of the dispersion law is observed in the MSSW geometry. The nonreciprocity is explained by unequal action of the image components of magnetostatic fields at opposite directions of wave propagation. Overall, this work emphasizes that coupling of spin waves with a superconductor provides a powerful practical tool for tuning their dispersion law.

\section{SUPPLEMENTARY MATERIAL}

See supplementary material for comparison of dispersions for several thicknesses of FM films at different magnetic fields.

\section{ACKNOWLEDGMENTS}

The authors acknowledge Professor S. O. Demokritov for fruitful discussions. This work was supported by the Russian Science Foundation (RSF) (Project No. 18-72-00224).

${ }^{1}$ F. Bloch, Z. Phys. 61, 206 (1930).

${ }^{2}$ R. W. Damon and J. R. Eshbach, J. Appl. Phys. 31, S104 (1960).

${ }^{3}$ D. Stancil, Theory of Magnetostatic Waves (Springer-Verlag, New York, 1993).

${ }^{4}$ Magnetization Oscillations and Waves, edited by A. G. Gurevich and G. A. Melkov (CRC Press, 1996).

5Magnonics: From Fundamentals to Applications, edited by S. O. Demokritov and A. N. Slavin (Springer-Verlag, Berlin, 2013).

${ }^{6}$ G. Csaba, A. Papp, and W. Porod, Phys. Lett. A 381, 1471 (2017).

${ }^{7}$ Y. Kajiwara, K. Harii, S. Takahashi, J. Ohe, K. Uchida, M. Mizuguchi, H. Umezawa, H. Kawai, K. Ando, K. Takanashi, S. Maekawa, and E. Saitoh, Nature 464, 262 (2010).

${ }^{8}$ A. V. Chumak, V. I. Vasyuchka, A. A. Serga, and B. Hillebrands, Nat. Phys. 11, 453 (2015).

${ }^{9}$ A. Haldar, C. Tian, and A. O. Adeyeye, Sci. Adv. 3, 1700638 (2017).

${ }^{10}$ A. Khitun, R. Ostroumov, and K. L. Wang, Phys. Rev. A 64, 062304 (2001).

${ }^{11}$ P. Andrich, C. F. de las Casas, X. Liu, H. L. Bretscher, J. R. Berman, F. J. Heremans, P. F. Nealey, and D. D. Awschalom, npj Quantum Inf. 3, 28 (2017).

${ }^{12}$ A. V. Chumak, A. A. Serga, and B. Hillebrands, Nat. Commun. 5, 4700 (2014).

${ }^{13}$ N. Sato, K. Sekiguchi, and Y. Nozaki, Appl. Phys. Express 6, 063001 (2013).

${ }^{14}$ M. Jamali, J. H. Kwon, S.-M. Seo, K.-J. Lee, and H. Yang, Sci. Rep. 3, 3160 (2013).

${ }^{15}$ A. A. Serga, A. V. Chumak, and B. Hillebrands, J. Phys. D Appl. Phys. 43, 264002 (2010).

${ }^{16} \mathrm{P}$. Gruszecki and M. Krawczyk, "Magnonic crystals," in Wiley Encyclopedia of Electrical and Electronics Engineering (John Wiley \& Sons, Ltd., 2016) 
${ }^{17}$ I. A. Golovchanskiy, N. N. Abramov, V. S. Stolyarov, V. V. Bolginov, V. V. Ryazanov, A. A. Golubov, and A. V. Ustinov, Adv. Funct. Mater. 28, 1802375 (2018)

${ }^{18} \mathrm{M}$. Donahue and D. Porter, OOMMF User's Guide, Version 1.0, Interagency Report NISTIR 6376, National Institute of Standards and Technology, Gaithersburg, MD, 1999.

${ }^{19}$ J. E. Miltat and M. J. Donahue, "Numerical micromagnetics: Finite difference methods," in Handbook of Magnetism and Advanced Magnetic Materials (John Wiley \& Sons, Ltd., 2007).

${ }^{20}$ P. Deorani, J. H. Kwon, and H. Yang, Curr. Appl. Phys. 14, S129 (2014).

${ }^{21}$ P. Wessels, A. Vogel, J.-N. Todt, M. Wieland, G. Meier, and M. Drescher, Sci. Rep. 6, 22117 (2016).

${ }^{22}$ T. Bracher, P. Pirro, J. Westermann, T. Sebastian, B. Lagel, B. V. de Wiele, A. Vansteenkiste, and B. Hillebrands, Appl. Phys. Lett. 102, 132411 (2013).

${ }^{23}$ V. Vlaminck and M. Bailleul, Phys. Rev. B 81, 014425 (2010).

${ }^{24}$ C. Liu, J. Chen, T. Liu, F. Heimbach, H. Yu, Y. Xiao, J. Hu, M. Liu, H. Chang, T. Stueckler, S. Tu, Y. Zhang, Y. Zhang, P. Gao, Z. Liao, D. Yu, K. Xia, N. Lei, W. Zhao, and M. Wu, Nat. Commun. 9, 738 (2018).

${ }^{25}$ A. Navabi, C. Chen, M. Aldosary, J. Li, K. Wong, Q. Hu, J. Shi, G. P. Carman, A. E. Sepulveda, P. K. Amiri, and K. L. Wang, Phys. Rev. Appl. 7, 034027 (2017).
${ }^{26}$ Y. V. Khivintsev, L. Reisman, J. Lovejoy, R. Adam, C. M. Schneider, R. E. Camley, and Z. J. Celinski, J. Appl. Phys. 108, 023907 (2010).

${ }^{27}$ B. V. de Wiele, S. J. Hamalainen, P. Balaz, F. Montoncello, and S. van Dijken, Sci. Rep. 6, 21330 (2016).

${ }^{28}$ G. Venkat, D. Kumar, M. Franchin, O. Dmytriiev, M. Mruczkiewicz, H. Fangohr, A. Barman, M. Krawczyk, and A. Prabhakar, IEEE Trans. Magn. 49, 524 (2013)

${ }^{29}$ F. S. Ma, H. S. Lim, Z. K. Wang, S. N. Piramanayagam, S. C. Ng, and M. H. Kuok, Appl. Phys. Lett. 98, 153107 (2011).

${ }^{30}$ I. A. Golovchanskiy, V. V. Bolginov, N. N. Abramov, V. S. Stolyarov, A. B. Hamida, V. I. Chichkov, D. Roditchev, and V. V. Ryazanov, J. Appl. Phys. 120, 163902 (2016).

${ }^{31}$ M. Mruczkiewicz and M. Krawczyk, J. Appl. Phys. 115, 113909 (2014).

${ }^{32}$ S. R. Seshadr, Proc. IEEE 58, 506 (1970).

${ }^{33}$ T. Yukawa, J. Yamada, K. Abe, and J. Ikenoue, Jpn. J. Appl. Phys. 16, 2187 (1977).

${ }^{34}$ I. A. Golovchanskiy, N. N. Abramov, V. S. Stolyarov, I. V. Shchetinin, P. S. Dzhumaev, A. S. Averkin, S. N. Kozlov, A. A. Golubov, V. V. Ryazanov, and A. V. Ustinov, J. Appl. Phys. 123, 173904 (2018).

${ }^{35}$ J. Lindner, K. Lenz, E. Kosubek, K. Baberschke, D. Spoddig, R. Meckenstock, J. Pelzl, Z. Frait, and D. L. Mills, Phys. Rev. B 68, 060102 (2003). 